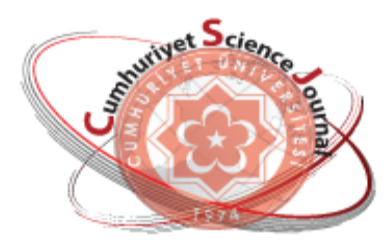

e-ISSN: 2587-246X

ISSN: $2587-2680$

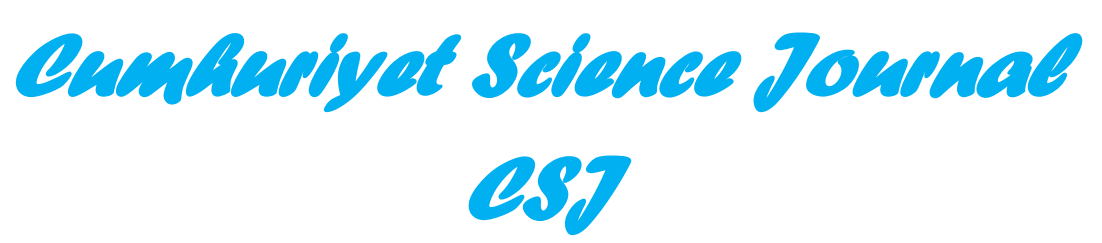

Cumhuriyet Sci. J., Vol.40-2 (2019) 369-377

\title{
On The Inverse Sum In Degree Index and Co Index
}

\section{Gülistan KAYA GÖK}

${ }^{1}$ Hakkari University, Department of Mathematics Education, Hakkari, TURKEY

\begin{abstract}
The inverse sum in degree index of $\mathrm{G}$ is specified the degrees $d_{i}$ and $d_{j}$. Some bounds are found for inverse sum in degree index in this study. Also, some definitions and relations are obtained in terms of degrees.

Keywords: İnverse sum in degree index, co index.
\end{abstract}

\section{Derece Endeksinde ve Ko Endeksinde Ters Toplam}

Özet. G' nin derece endeksinde ters toplam $d_{i}$ ve $d_{j}$ dereceleri ile belirtilir. Bu çalışmada, derece endeksinde toplam için bazı sınırlar bulunur. Ayrıca, dereceler açısından bazı tanımlar ve bağıntılar elde edilir.

Anahtar Kelimeler: Derece endeksinde ters toplam, Eş endeks.

\section{INTRODUCTION}

Let $\mathrm{G}$ be a simple, connected graph on the vertex set $\mathrm{V}(\mathrm{G})$ and the edge set $\mathrm{E}(\mathrm{G})$. For $v_{i} \in V(G)$, the degree of vertex $v_{i}$ denoted by $d_{i}$, the maximum degree is denoted by $\Delta$ and the minimum degree is denoted by $\delta$.

The inverse sum in degree matrix $[I S I](G)$ of graphs is defined as

$$
[I S I]_{i j}=\left\{\begin{array}{cc}
\frac{d_{i}+d_{j}}{d_{i} d_{j}} & \text { if } i \text { adjacent to } j \\
0 & \text { otherwise. }
\end{array}\right.
$$

The eigenvalues of $[I S I](G)$ are denoted by $\delta^{+}$. New bounds for these eigenvalues are reported in terms of the degrees.

The Inverse Sum In Degree Index (ISI) index of G is defined as

$$
\sum_{v_{i} v_{j} \in E(G)} \frac{d_{i}+d_{j}}{d_{i} d_{j}}
$$

(See [2] for details.)

In this study, different bounds are set using the Estrada index and Zagreb index for ISI index. 
The Estrada index of graph $\mathrm{G}$ is explained as

$$
E E(G)=\sum_{i=1}^{n} e^{\lambda_{i}}
$$

where $\lambda$ is the eigenvalue of adjacency matrix of G. ( [1], [10])

The Zagreb co index of $\mathrm{G}$ is described in [5], [7] as

$$
\begin{gathered}
\overline{Z_{1}}(G)=\sum_{v_{i} v_{j} \notin E(G),}\left(d_{G}(i)+d_{G}(j)\right), \\
\overline{Z_{2}}(G)=\sum_{v_{i} v_{j} \notin E(G),}\left(d_{G}(i) d_{G}(j)\right) .
\end{gathered}
$$

The Harmonic index of $\mathrm{G}$ is specified in [8] as

$$
H(G)=\sum_{v_{i} v_{j} \in E(G)} \frac{2}{d_{i}+d_{j}}
$$

Considering these topological indices, Estrada inverse sum in degree index and inverse sum in degree co index are defined. Indeed, some inequalities are obtained concerned with these indices.

(See [6] for more details deal with this topic.)

\section{PRELIMINARIES}

In this section, some lemmas and theorems that are needed in main results will be given.

\section{Lemma 2.1. [9]}

Let $M=\left(m_{i j}\right)$ be an $n x n$ irreducible nonnegative matrix and $\lambda_{1}(M)$ be the greatest eigenvalue with $R_{i}(M)=\sum_{j=1}^{m} m_{i j}$. Then,

$$
\left(\min _{i}(M): 1 \leq i \leq n\right) \leq \lambda_{1}(M) \leq\left(\max R_{i}(M): 1 \leq i \leq n\right)
$$

\section{Lemma 2.2. [4]}

If $G$ is a simple connected graph and $\lambda_{1}(G)$ is the spectral radius, then

$$
\lambda_{1}(G) \leq \max \left(\sqrt{m_{i} m_{j}}: 1 \leq i, j \leq n, v_{i}, v_{j} \in E\right)
$$




\section{Theorem 2.1. [3]}

If $a_{i}, b_{i} \in R^{+}, 1 \leq i \leq n$, then

$$
\sum_{i=1}^{n} a_{i}{ }^{2} \sum_{i=1}^{n} b_{i}{ }^{2}-\left(\sum_{i=1}^{n} a_{i} b_{i}\right)^{2} \leq \frac{n^{2}}{4}\left(M_{1} M_{2}-m_{1} m_{2}\right)^{2}
$$

where $M_{1}=\max _{1 \leq i \leq n} a_{i}, M_{2}=\max _{1 \leq i \leq n} b_{i}, m_{1}=\min _{1 \leq i \leq n} a_{i}, m_{2}=\min _{1 \leq i \leq n} b_{i}$.

Theorem 2.2. [5]

Let $G$ be a graph with $n$ vertices and $m$ edges. Then,

$$
\begin{gathered}
Z_{1}(\bar{G})=Z_{1}(G)+n(n-1)^{2}-4 m(n-1) \\
\overline{Z_{1}}(G)=2 m(n-1)-Z_{1}(G)=\overline{Z_{1}}(\bar{G}) .
\end{gathered}
$$

Lemma 2.3. [7]

If $G$ is a regular graph, then

$$
\begin{gathered}
Z_{1}(G) \geq \frac{4 m^{2}}{n}, \\
Z_{2}(G) \geq \frac{4 m^{3}}{n^{2}}, \\
\overline{Z_{1}}(G) \leq \frac{-4 m^{2}}{n}+2 m(n-1), \\
\overline{Z_{2}}(G) \leq 2 m^{2}\left(1-\frac{2 m}{n^{2}}-\frac{1}{n}\right) .
\end{gathered}
$$

\section{MAIN RESULTS}

\subsection{Inverse Sum In Degree Index and Estrada Inverse Sum In Degree Index}

Firstly, a relation is given for the largest eigenvalue of ISI matrix including degrees in this subsection. After, an inequality is obtained for ISI index using this relation. In addition, Estrada inverse sum in degree index is defined and some relations are found in terms of degrees and vertices.

Theorem 3.1.1. Let $G$ be graph on $n$ vertices and $m$ edges. Then,

$$
I S I(G) \geq \sqrt{\left(Z_{2}(G) H(G)\right)^{2}-\frac{n^{2}}{4}\left(\frac{\Delta^{3}-\delta^{3}}{2 \Delta \delta}\right)} .
$$

Proof. Let choose $a_{k}=d_{i} d_{j}, b_{k}=\frac{1}{d_{i}+d_{j}}, M_{1}=\Delta^{2}, m_{1}=\delta^{2}, M_{2}=\frac{1}{2 \delta}, m_{2}=\frac{1}{2 \Delta}$.

By Theorem 2.1, it is seen that 


$$
\sum_{v_{i} v_{j} \in E(G)}\left(d_{i} d_{j}\right)^{2} \sum_{v_{i} v_{j} \in E(G)}\left(\frac{1}{d_{i}+d_{j}}\right)^{2}-\left(\sum_{v_{i} v_{j} \in E(G)} \frac{d_{i} d_{j}}{d_{i}+d_{j}}\right)^{2} \leq \frac{n^{2}}{4}\left(\frac{\Delta^{2}}{2 \delta}-\frac{\delta^{2}}{2 \Delta}\right)
$$

If necessary organizing is applied, this inequality is obtained as follows:

$$
\begin{gathered}
\left(\sum_{v_{i} v_{j} \in E(G)}\left(d_{i} d_{j}\right)\right)^{2}\left(\sum_{v_{i} v_{j} \in E(G)}\left(\frac{1}{d_{i}+d_{j}}\right)\right)^{2}-\frac{n^{2}}{4}\left(\frac{\Delta^{2}}{2 \delta}-\frac{\delta^{2}}{2 \Delta}\right) \\
\leq\left(\sum_{v_{i} v_{j} \in E(G)} \frac{d_{i} d_{j}}{d_{i}+d_{j}}\right)^{2} .
\end{gathered}
$$

Putting the definitions in the above inequality, it gets

$$
\left(Z_{2}(G)\right)^{2}(H(G))^{2}-\frac{n^{2}}{4}\left(\frac{\Delta^{3}-\delta^{3}}{2 \Delta \delta}\right) \leq(I S I(G))^{2} .
$$

Hence,

$$
I S I(G) \geq \sqrt{\left(Z_{2}(G) H(G)\right)^{2}-\frac{n^{2}}{4}\left(\frac{\Delta^{3}-\delta^{3}}{2 \Delta \delta}\right)} .
$$

Lemma 3.1.1. For a simple connected graph of $I S I(G)$,

$$
\gamma_{1}^{+} \leq \frac{\Delta}{n^{1 / n} \sqrt{\left(d_{i}^{n}+\Delta\right)\left(d_{j}^{n}+\Delta\right)}}
$$

where $\Delta$ is the maximum degree of $G$.

Proof. Let $D(G)^{-1} \operatorname{ISI}(G) D(G)=Q(G)$ and $X=\left(x_{1}, x_{2}, \ldots, x_{n}\right)^{T}$ be an eigen vector of $Q(G)$ corresponding to an eigen value $\gamma^{+}$. Also, $x_{i}=1$ and $0<x_{k} \leq 1$ for every $k$. Let $x_{j}=$ $\max _{k}\left(x_{k}: v_{i} v_{k} \in E, i\right.$ is adjacent to $\left.k\right)$. It is known that $(Q(G)) X=\gamma_{1}^{+}(G) X$. If $i_{-}$th equation from above equation is taken, then $\gamma_{1}^{+}(G) x_{i}=\sum_{k}\left(\frac{d_{i} d_{k}}{d_{i}+d_{k}}\right) x_{k}=\left(d_{i} \sum_{k} \frac{d_{k}}{d_{i}+d_{k}}\right) x_{k}$. By the Aritmetic-Geometric mean inequality, it gives 


$$
\begin{gathered}
\frac{\sum_{k} \frac{d_{k}}{d_{i}+d_{k}}}{n} \geq\left(\frac{\prod_{k=1}^{n} \frac{d_{k}}{d_{i}+d_{k}}}{n}\right)^{1 / n} \geq \frac{\left(\frac{\prod_{k=1}^{n} d_{k}}{\prod_{k=1}^{n}\left(d_{i}+d_{k}\right)}\right)^{1 / n}}{n^{1 / n}} \\
\geq \frac{\frac{\left(\Delta^{n}\right)^{1 / n}}{\left(d_{i}^{n}+\prod_{k=1}^{n} d_{k}\right)^{1 / n}}}{n^{1 / n}} \geq \frac{\Delta}{n^{1 / n}\left(d_{i}^{n}+\left(\Delta^{n}\right)^{1 / n}\right)} .
\end{gathered}
$$

Using the Lemma 2.1,

$$
\gamma_{1}^{+}(G) \leq \frac{d_{i} \Delta}{n^{1 / n}\left(d_{i}^{n}+\Delta\right)}
$$

The $j \_$th equation of same equation has

$$
\gamma_{1}^{+}(G) \leq \frac{d_{j} \Delta}{n^{1 / n}\left(d_{j}^{n}+\Delta\right)}
$$

From Lemma 2.2, it is expressed that

$$
\gamma_{1}^{+}(G) \leq \sqrt{\left(\frac{d_{i} \Delta}{n^{1 / n}\left(d_{i}^{n}+\Delta\right)}\right)\left(\frac{d_{j} \Delta}{n^{1 / n}\left(d_{j}^{n}+\Delta\right)}\right)} .
$$

Hence,

$$
\gamma_{1}^{+} \leq \frac{\Delta}{n^{1 / n} \sqrt{\left(d_{i}^{n}+\Delta\right)\left(d_{j}^{n}+\Delta\right)}}
$$

Since, $\Delta=d_{1} \geq d_{2} \geq, \ldots, \geq d_{n}=\delta$, it is clear that

$$
\gamma_{1}^{+} \leq \frac{\Delta}{n^{1 / n} \sqrt{\left(\Delta^{n}+\Delta\right)\left(\delta^{n}+\Delta\right)}}
$$

Definition 3.1.1. Let $G$ be a graph and $\gamma_{1}^{+} \geq \gamma_{2}^{+} \geq \cdots \geq \gamma_{n}^{+}$be eigenvalues of inverse sum in degree matrix of $G$. Estrada inverse sum in degree index is defined as

$$
E_{\iota S l}=\sum_{j=1}^{n} e^{\gamma_{j}^{+}}
$$

Theorem 3.1.2. Let $G$ be a graph with $n$ vertices and $E_{l s l}$ be an Estrada inverse sum in deg index. Then,

$$
E_{l s l} \geq e^{K}+\frac{(n-1)}{e^{1 / n-1}}
$$


where $K=\frac{\Delta}{n^{1 / n} \sqrt{\left(\Delta^{n}+\Delta\right)\left(\delta^{n}+\Delta\right)}}$.

Proof. $E_{l s l}=\sum_{j=1}^{n} e^{\gamma_{j}^{+}} \geq e^{\gamma_{1}^{+}}+(n-1)\left(\prod_{J=2}^{n} e^{\gamma_{J}^{+}}\right)^{1 / n-1}$ using the Aritmetic-Geometric mean inequality. Since, $\sum_{i=1}^{n} e^{\gamma_{J}^{+}}=0$ then $E_{l s l} \geq e^{\gamma_{J}^{+}}+\frac{(n-1)}{e^{1 / n-1}}$. It is known that $\gamma_{J}^{+} \leq K$. Hence,

$$
E_{l s l} \geq e^{K}+\frac{(n-1)}{e^{1 / n-1}}
$$

Theorem 3.1.3. Let $G$ be a graph with $n$ vertices and $E_{l s l}$ be an Estrada inverse sum in deg. index. Then,

$$
E_{l s l} \leq \sqrt{-K n \sum_{k \geq 0}^{\infty} \frac{2^{k}}{k !}}
$$

Proof. It is easy to see that $\frac{1}{n} \sum_{j=1}^{n}\left(e^{\gamma_{j}^{+}}\right)^{2} \geq\left(\frac{1}{n} \sum_{j=1}^{n} e^{\gamma_{j}^{+}}\right)^{2}$. On the other hand, $\frac{1}{n} \sum_{j=1}^{n} e^{2 \gamma_{j}^{+}} \geq$ $\frac{1}{n^{2}} E_{l s l}^{2}$. Hence,

$$
\text { n. } \sum_{k \geq 0}^{\infty} \frac{1}{k !} \sum_{j=1}^{n}\left(2 \gamma_{j}^{+}\right)^{k} \geq E_{l s l}{ }^{2}
$$

and thus,

$$
E_{l s l}{ }^{2} \leq n . \sum_{k \geq 0}^{\infty} \frac{2^{k}}{k !} \sum_{j=1}^{n}\left(\gamma_{j}^{+}\right)^{k}
$$

Knowing that $\gamma_{1}^{+} \geq \cdots \geq \gamma_{n}^{+}$and $\gamma_{1}^{+} \leq K$, it is obtained that

$$
E_{l s l}^{2} \leq n . \sum_{k \geq 0}^{\infty} \frac{2^{k}}{k !} \cdot n \cdot K^{k} .
$$

It is clear that the equality holds

$$
E_{l S l} \leq \sqrt{n^{2} \cdot \sum_{k \geq 0}^{\infty} \frac{(2 K)^{k}}{k !}} .
$$

Theorem 3.1.4. Let $G$ be a graph with $n$ vertices and $E_{l s l}$ be an Estrada inverse sum in degree index. Then,

$$
E_{l s l} \leq \sqrt{e^{2 K}-2 e^{K}}+e^{K} .
$$


Proof. $\left(E_{l s l}-e^{\gamma_{1}^{+}}\right)^{2}=\left(\sum_{j=1}^{n} e^{\gamma_{j}^{+}}\right)^{2}-2\left(\sum_{j=1}^{n} e^{\gamma_{j}^{+}} e^{\gamma_{1}^{+}}\right)+e^{2 \gamma_{1}^{+}}$

$$
\leq\left(\sum_{j=1}^{n} e^{\gamma_{j}^{+}}\right)^{2}-2 n e^{\gamma_{1}^{+}}\left(\prod_{j=1}^{n} e^{\gamma_{j}^{+}}\right)^{1 / n}+e^{2 \gamma_{1}^{+}}
$$

Since $\left(\sum_{j=1}^{n} e^{\gamma_{j}^{+}}\right)^{2}=\left(\sum_{k \geq 0}^{\infty} \frac{1}{k !} \sum_{j=1}^{n}\left(\gamma_{j}^{+}\right)^{k}\right)^{2} \leq\left(\sum_{k \geq 0}^{\infty} \frac{1}{k !}\left(\sum_{j=1}^{n} \gamma_{j}^{+}\right)^{k}\right)^{2}$ and $\sum_{j=1}^{n} \gamma_{j}^{+}=0$, then

$$
\left(E_{l s l}-e^{\gamma_{1}^{+}}\right)^{2} \leq-2 n e^{\gamma_{1}^{+}}+e^{2 \gamma_{1}^{+}}=e^{\gamma_{1}^{+}}\left[e^{\gamma_{1}^{+}}-2 n\right] .
$$

The inequality states that

$$
E_{l s l} \leq \sqrt{e^{\gamma_{1}^{+}}\left(e^{\gamma_{1}^{+}}-2 n\right)}+e^{\gamma_{1}^{+}}
$$

In the sequel, Theorem 3.1.2 says that

$$
E_{l S l} \leq \sqrt{e^{K}\left(e^{K}-2 n\right)}+e^{K} .
$$

\subsection{Inverse Sum In Degree Co Index}

In this subsection, ISI co index is described and different bounds are yielded concerned with Zagreb co indices, the vertices and the edges.

\section{Definition 3.2.1.}

Let $G$ be a simple, connected graph. ISI co index is defined as follows:

$$
I S I(\bar{G})=\sum_{v_{i} v_{j} \notin E(G)} \frac{d_{G}(i) d_{G}(j)}{d_{G}(i)+d_{G}(j)} .
$$

Theorem 3.2.1. Let $\operatorname{ISI}(\bar{G})$ be the complement of inverse sum in degree index. If $\mathrm{G}$ is regular then,

$$
I S I(\bar{G}) \leq \frac{(n-1)^{2}\left[\left(\begin{array}{l}
n \\
2
\end{array}\right)-m\right]-(n-1) \cdot\left(-\frac{4 m^{2}}{n}+2 m(n-1)\right)+\left(2 m^{2}\left(1-\frac{2 m}{n^{2}}-\frac{1}{n}\right)\right)}{2 \cdot(n-1) \cdot\left[\left(\begin{array}{l}
n \\
2
\end{array}\right)-m\right]+\frac{4 m^{2}}{n}-(2 m(n-1))} .
$$

Proof. By the definition of $I S I(\bar{G}) ; I S I(\bar{G})=\sum_{v_{i} v_{j} \in E(\bar{G})} \frac{d_{\bar{G}}(i) d_{\bar{G}}(j)}{d_{\bar{G}}(i)+d_{\bar{G}}(j)}$. Since $d_{\bar{G}}(i)=(n-1-$ $\left.d_{i}\right)$ and $d_{\bar{G}}(j)=\left(n-1-d_{j}\right)$, then 


$$
\begin{gathered}
I S I(\bar{G})=\sum_{v_{i} v_{j} \in E(\bar{G})} \frac{\left(n-1-d_{i}\right) \cdot\left(n-1-d_{j}\right)}{\left(n-1-d_{i}\right)+\left(n-1-d_{j}\right)} \\
=\sum_{v_{i} v_{j} \in E(\bar{G})} \frac{(n-1)^{2}-(n-1)\left(d_{i}+d_{j}\right)+d_{i} d_{j}}{2(n-1)-\left(d_{i}+d_{j}\right)} \\
\leq \frac{\sum_{v_{i} v_{j} \in E(\bar{G})}(n-1)^{2}-(n-1)\left(d_{i}+d_{j}\right)+d_{i} d_{j}}{\sum_{v_{i} v_{j} \in E(\bar{G})} 2(n-1)-\left(d_{i}+d_{j}\right)} \\
I S I(\bar{G}) \leq \frac{(n-1)^{2} \sum_{v_{i} v_{j} \in E(\bar{G})} 1-(n-1) \sum_{v_{i} v_{j} \in E(\bar{G})}\left(d_{i}+d_{j}\right)+\sum_{v_{i} v_{j} \in E(\bar{G})} d_{i} d_{j}}{2 \cdot(n-1) \cdot\left[\left(\begin{array}{l}
n \\
2
\end{array}\right)-m\right]-\sum_{v_{i} v_{j} \in E(\bar{G})}\left(d_{i}+d_{j}\right)} .
\end{gathered}
$$

Because, G has $\left(\begin{array}{l}n \\ 2\end{array}\right)-m$ edges. By Theorem 2.2, it is stated that

$$
I S I(\bar{G}) \leq \frac{(n-1)^{2}\left[\left(\begin{array}{l}
n \\
2
\end{array}\right)-m\right]-(n-1) Z_{1}(\bar{G})+Z_{2}(\bar{G})}{2 \cdot(n-1) \cdot\left[\left(\begin{array}{l}
n \\
2
\end{array}\right)-m\right]-Z_{1}(\bar{G})}
$$

Since $Z_{1}(\bar{G})=\overline{Z_{1}}(G)$, then

$$
I S I(\bar{G}) \leq \frac{(n-1)^{2}\left[\left(\begin{array}{l}
n \\
2
\end{array}\right)-m\right]-(n-1) \cdot \overline{Z_{1}}(G)+\overline{Z_{2}}(G)}{2 \cdot(n-1) \cdot\left[\left(\begin{array}{l}
n \\
2
\end{array}\right)-m\right]-\overline{Z_{1}}(G)} .
$$

Using the Lemma 2.3, it is concluded that

$$
I S I(\bar{G}) \leq \frac{(n-1)^{2}\left[\left(\begin{array}{l}
n \\
2
\end{array}\right)-m\right]-(n-1) \cdot\left(-\frac{4 m^{2}}{n}+2 m(n-1)\right)+\left(2 m^{2}\left(1-\frac{2 m}{n^{2}}-\frac{1}{n}\right)\right)}{2 \cdot(n-1) \cdot\left[\left(\begin{array}{l}
n \\
2
\end{array}\right)-m\right]+\frac{4 m^{2}}{n}-(2 m(n-1))} .
$$

Corollary 3.2.1. Let $I S I(\bar{G})$ be the complement of inverse sum in degree index. If $\mathrm{G}$ is regular then,

$$
\overline{I S I}(\bar{G}) \leq \frac{(n-1) m-(n-1) \cdot\left(-\frac{4 m^{2}}{n}+2 m(n-1)\right)+\left(2 m^{2}\left(1-\frac{2 m}{n^{2}}-\frac{1}{n}\right)\right)}{2 \cdot(n-1) m+\frac{4 m^{2}}{n}-(2 m(n-1))}
$$

Proof. Applying similar steps to the Theorem 3.2.1, it is obtained that

$$
\begin{aligned}
\overline{I S I}(\bar{G}) & =\sum_{v_{i} v_{j} \notin E(\bar{G})} \frac{d_{\bar{G}}(i) \cdot d_{\bar{G}}(j)}{d_{\bar{G}}(i)+d_{\bar{G}}(j)} \\
& =\sum_{v_{i} v_{j} \in E(G)} \frac{(n-1)^{2}-(n-1)\left(d_{i}+d_{j}\right)+d_{i} d_{j}}{2(n-1)-\left(d_{i}+d_{j}\right)}
\end{aligned}
$$




$$
\leq \frac{(n-1)^{2} m-(n-1) \cdot \overline{Z_{1}}(G)+\overline{Z_{2}}(G)}{2 \cdot(n-1) m-\overline{Z_{1}}(G)}
$$

By Lemma 2.3, it is resulted that

$$
\overline{I S I}(\bar{G}) \leq \frac{(n-1)^{2} m-(n-1) \cdot\left(-\frac{4 m^{2}}{n}+2 m(n-1)\right)+\left(2 m^{2}\left(1-\frac{2 m}{n^{2}}-\frac{1}{n}\right)\right)}{2 .(n-1) m+\frac{4 m^{2}}{n}-(2 m(n-1))}
$$

\section{CONCLUSION}

In this study, the inverse sum in degree index is expanded, the Estrada inverse sum in degree index is defined and some bounds are found deal with these indices. In the sequel, inverse sum in degree co index is described and some inequalities are obtained in terms of the edges and vertices.

\section{ACKNOWLEDGEMENT}

The author would like thank for the valuable suggestions of referees.

\section{REFERENCES}

[1] Estrada E., Characterization of 3D molecular structure, Chem. Phys. Lett., 319 (2000) 713-718.

[2] Sedlar J., Stevanović D. and Vasilyev A., On the inverse sum in degree index, Discrete Applications Mathematics, 184 (2015) 202-212.

[3] Kaya Gök G., Some bounds on the distance-sum-connectivity matrix, Journal of Inequalities and Applications, (2018) 171.

[4] Das K. C. and Kumar P., Some new bounds on the spectral radius of graphs, Discrete Mathematics, 281 (2004) 149-161.

[5] Gutman I., Furtula B., Vukićević Z.V. and Popivoda G., On Zagreb Indices and Co indices, MATCH Commun. Math. Comput. Chem., 74 (2015) 5-16.

[6] Sorgun S. and Büyükköse Ş., The new upper bounds on the spectral radius of weighted graphs, Applied Mathematics and Computation, 218 (2012) 5231-5238.

[7] Hossein-Zadeh S., Hamzeh A. and Ashrafi A. R., Extremal Properties of Zagreb Co indices and Degree Distance of Graphs, Miskolc Mathematical Notes, 11-2 (2010) 129-137.

[8] Fajtlowicz S., On conjectures on Graffiti-II, Congr. Number, 60 (1987) 187-197.

[9] Horn R.A. and Johnson C.R., Matrix Analysis: Cambridge University Press, New York, 1985.

[10] Du Z. and Zhongzhu L., On the Estrada and Laplacian Estrada indices of graphs, Linear Algebra and its Applications, 435 (2011) 2065-2076. 